\title{
BMJ Open Patterns of relapse in extrapulmonary small cell carcinoma: retrospective analysis of outcomes from two cancer centres
}

\author{
S Gennatas, ${ }^{1,2}$ J Noble, ${ }^{2}$ S Stanway, ${ }^{2}$ R Gunapala, ${ }^{2}$ R Chowdhury, ${ }^{3}$ \\ A Wotherspoon, ${ }^{4}$ T Benepal, ${ }^{5}$ S Popat ${ }^{1,2}$
}

To cite: Gennatas S, Noble J, Stanway S, et al. Patterns of relapse in extrapulmonary small cell carcinoma: retrospective analysis of outcomes from two cancer centres. BMJ Open 2015;5: 006440. doi:10.1136/bmjopen-2014006440

- Prepublication history for this paper is available online. To view these files please visit the journal online (http://dx.doi.org/10.1136/ bmjopen-2014-006440).

Received 22 August 2014 Revised 15 December 2014 Accepted 18 December 2014

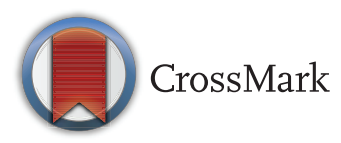

\footnotetext{
${ }^{1}$ Faculty of Medicine, National Heart and Lung Institute, Imperial College London, London, UK

${ }^{2}$ Department of Medicine, Royal Marsden Hospital, London, UK

${ }^{3}$ Department of Medicine, Dimbleby Cancer Centre, Kings College London,

London, UK

${ }^{4}$ Department of Histopathology, Royal Marsden Hospital, London, UK

${ }^{5}$ Department of Oncology, St George's Hospital, London, UK
}

Correspondence to Dr S Popat; sanjay.popat@rmh.nhs.uk

\section{ABSTRACT}

Objectives: We conducted a retrospective review of patients with extrapulmonary small cell carcinomas (EPSCCs) to explore the distribution, treatments, patterns of relapse and outcomes by primary site.

Setting: We have reviewed the outcomes of one of the largest data sets of consecutive patients with EPSCC identified from two major cancer centres.

Participants: Consecutive patients with a histopathological diagnosis of EPSCC from the two institutions were retrospectively identified.

Primary and secondary outcome measures:

Outcomes were evaluated including stage at presentation, treatments given, sites of relapse, time to distant relapse, progression-free survival and overall survival (OS).

Results: From a total 159 patients, 114 received firstline chemotherapy, $80.5 \%$ being platinum-based. Response rate was $48 \%$. Commonest primary sites were genitourinary and gynaecological. $44 \%$ of patients presented with metastatic disease. $55.9 \%$ relapsed with liver the commonest site, whereas only $2.5 \%$ developed brain metastases. Median OS was 13.4 months for all patients, 7.6 months and 19.5 months for those with metastatic and nonmetastatic disease, respectively. Gynaecological and head and neck patients had significantly better OS compared to gastrointestinal patients.

Conclusions: EPSCCs demonstrate high response rates to chemotherapy and high rates of distant metastases. Primary sites may influence prognosis, and survival is optimal with a radical strategy. Brain metastases are rare and we therefore do not recommend prophylactic cranial irradiation.

\section{INTRODUCTION}

Neuroendocrine tumours are epithelial neoplasms with predominant neuroendocrine differentiation and, while typically seen of pulmonary origin, can arise in most organs. ${ }^{1}$ Pathological classification is contingent on site of origin, ranging from low-grade

\section{Strengths and limitations of this study}

- This is a retrospective study on one of the largest consecutive patient series reported with extrapulmonary small cell carcinomas (EPSCC).

- The outcomes of this study are consistent with data from other, smaller data sets.

- The study highlights significant findings on a variety of EPSCC outcomes, including response to chemotherapy and rate of metastatic disease, including brain metastases, according to primary site.

- Observed differences in outcomes by site are influenced by numbers of cases of each anatomical location identified, which in turn likely reflects local referral patterns.

Lack of central pathology verification.

carcinoid tumours to high-grade carcinomas, and outside the lung, the WHO classification broadly divides them into three main grades (1-3), with grade 3 tumours the classifier for neuroendocrine carcinomas including extrapulmonary small cell carcinoma. ${ }^{12}$

Neuroendocrine carcinomas are most commonly of lung origin, typified by small cell lung cancer (SCLC), ${ }^{3}$ now representing around $13 \%$ of all lung cancer cases. ${ }^{4}$ Most patients have a history of smoking, ${ }^{5}$ and around $66 \%$ of patients present with metastatic (extensive stage) disease. ${ }^{3}$ Prognosis is poor, with a median overall survival (OS) of 2-4 months without treatment, ${ }^{3}$ rising to around 10 months, and a 2-year survival of $4.6 \%$ with chemotherapy. ${ }^{4}{ }^{7}$ Brain is a common site of metastatic disease, occurring in over $18 \%$ of patients at presentation, and up to $80 \%$ at 2 years. ${ }^{8}$ Patients with SCLC with localised disease may benefit from prophylactic cranial irradiation (PCI), with a higher progression free survival (PFS) (relative risk $(\mathrm{RR})=0.75,95 \%$ CI 0.65 to 0.86 , $\mathrm{p}<0.001)$ and $\mathrm{OS}(15.3 \%$ in the control 
group vs $20.7 \%$ in the PCI group at 3 years). It also decreases the risk of developing brain metastases $(\mathrm{RR}=0.46,95 \%$ CI 0.38 to $0.57, \mathrm{p}<0.001) .{ }^{9}$ In patients with extensive SCLC, PCI has been shown to significantly increase OS (HR $0.68 ; 95 \%$ CI 0.52 to 0.88 ) and significantly decrease risk of symptomatic brain metastases (from $40.4 \%$ to $14.6 \%$ at 1 year). ${ }^{8}$

Extrapulmonary small cell carcinomas (EPSCC) are rare high-grade neuroendocrine carcinomas arising outside the lungs, initially described in $1930 .^{10}$ Since the 1970 s, various descriptions including 'oat cell' and 'extrapulmonary oat cell carcinoma' have been used to describe EPSCC, a term that first came into use in the $1990 \mathrm{~s},{ }^{11}{ }^{12}$ to describe all small cell carcinomas arising outside the lungs. These account for $0.1-0.4 \%$ of all cancers and $2.5-5 \%$ of all small cell carcinomas in the USA. ${ }^{13}$ Since being described as a distinct entity, EPSCC has been identified from almost every body site excluding only the central nervous system (CNS). ${ }^{12} 1415$ Morphology, immunohistochemistry and ultrastructure are identical to SCLC and, while data are limited, potentially share common molecular features with SCLC, and also carcinomas that typically arise from each primary site. ${ }^{16}$ Given their rarity, most data sets are either case-reports or small patient series. These have suggested a poor $\mathrm{OS}^{14}$ and also suggested potential differences in patterns of relapse and outcome of EPSCC from differing primary sites, with breast, genitourinary, gynaecological, and head and neck tumours potentially more likely to present with localised disease, whereas gastrointestinal (GI) EPSCC is most likely metastatic. ${ }^{13} 141718$ Optimal chemotherapy is unknown due to data paucity, and EPSCC management is largely based on the SCLC paradigm utilising platinum-etoposide-based chemotherapy with or without radiotherapy. ${ }^{11} \quad 13-15 \quad 17 \quad{ }^{19-22}$ Series have been conflicting on incidence of brain metastases in EPSCC, some suggesting rates potentially lower than those in SCLC. 202324

We therefore aimed to retrospectively review consecutive cases of patients with EPSCC seen at two cancer centres, in order to determine the anatomical distribution at presentation, treatments, patterns of relapse and to explore differences in outcomes by site of primary.

\section{MATERIALS AND METHODS}

Patients were identified if registered at two neighbouring cancer centres within the South West London Cancer Network: The Royal Marsden Hospital and St George's Hospital. Eligible patients were those aged $\geq 18$, identified to have a diagnosis of small cell carcinoma including mixed subtypes (eg, adenocarcinoma/small cell carcinoma), but excluding those known to have a lung primary. Patients were identified from institutional pathology databases, electronic and paper-based patient records. Patients were recruited if registered at each institution up to April 2010, to allow for mature survival data. The study was classified and approved as a Service Evaluation at both institutions.
Data were collected in a common secured database with anonymised identifiers. Data points collected included: age, sex, gender, smoking history (never, current, ex-, unknown), diagnosis date, histological diagnosis, site of primary (subgrouped into breast, gynaecological, genitourinary, upper/lower GI, head and neck, other, unknown), performance status (at diagnosis and at each therapy point), stage at diagnosis (metastatic/nonmetastatic, radically/non-radically treatable), chemotherapy administered (regime, dates, best response), radiotherapy details (site, dose, fractionation, best response), surgery details (margin completeness), relapse dates, sites of relapse (locoregional/distant), treatment of relapse, date of death or last follow-up (and disease status). Individual pathology specimens were not centrally reviewed. Data were verified by one of the investigators (SG) in $10 \%$ of cases. No discrepancies were identified.

Descriptive statistics were used to summarise patient characteristics. OS was measured from date of diagnosis until death from any cause or censored at last follow-up date and calculated using the Kaplan-Meier method. Multivariate Cox regression was performed to assess influence of covariates. A forward stepwise selection process was used to build a multivariable model for OS. All variables with $p$ value $<0.2$ significance in the univariate analysis were included in the multivariate analysis to identify independent prognostic factors. For site of primary cancer, the Cox regression coefficients were determined relative to the reference category (arbitrarily defined as GI patients). PFS was measured from date of diagnosis until the first documented progression in any site following initial treatment or until death from any cause or censored at last follow-up date. Time to distant relapse (TTDR) was measured from date of last treatment received until date of first relapse or else censored at the date of last follow-up.

\section{RESULTS}

Between 05/05/1978 and 08/04/2010 data for 166 patients with a diagnosis of EPSCC were recorded between the two institutions. However, five patients were duplicates (due to hospital transfer) and two patients were less than 18 years old at diagnosis. Hence, only 159 patients were assessed for analysis. Mean age at diagnosis was 61 years ranging from 19 to 90 , with 70 males and 89 females (male: female ratio 1:1.3). Performance status at diagnosis was poorly documented (unknown for $75 \%$ of cases) as was weight loss (unknown for $72 \%$ of cases) and was, therefore, not included in analysis. Although smoking status was unknown for $48 \%$ of cases, in those with known status, only $13 \%$ and $58 \%$ of patients were current or ex-smokers at time of diagnosis, respectively.

The majority of cases were reported as pure EPSCC (123 cases, $77.4 \%$ ), while the remainder were admixed with other histological subtypes, including EPSCC/ 
adenocarcinoma $(18,11.3 \%)$, EPSCC/transitional cell carcinoma (12, 7.5\%), EPSCC/squamous cell carcinoma $(5,3.1 \%)$ and EPSCC/other (1, 0.6\%).

One hundred fourteen patients received chemotherapy alone or in combination with radiotherapy as firstline treatment. Response assessment data were available in 113 patients $(71.1 \%)$. Of those, $25(22.1 \%)$ were non-evaluable. In the 88 remaining patients, complete remission was observed in 6 patients $(6.8 \%)$, partial remission in $49(55.7 \%$ ) (Overall response rate was $62.5 \%$ in the 88 patients and $48 \%$ in all 114 patients), stable disease rate in $14(15.9 \%)$ and progression observed in 19 (21.6\%).

Of the 113 patients who received chemotherapy, 91 $(80.5 \%)$ received platinum-based chemotherapy (carboplatin or cisplatin), either alone or in combination. The commonest combination was carboplatin/etoposide doublet (37 patients, 32.8\% of all patients who received chemotherapy). In total, 71 patients $(62.8 \%)$ received combination chemotherapy containing etoposide. In 54 cases it was administered as part of a platinum-based doublet or triplet and in 16 as part of ACE (doxorubicin, cyclophosphamide, etoposide), which was the commonest non-platinum containing regimen. 65 of the 113 patients, who received chemotherapy, relapsed $(57.5 \%)$. Of those, $28(43.1 \%)$ received second-line chemotherapy. Of the 13 regimens given, the commonest was ACE (6 cases, 21.4\%). Ten patients $(35.7 \%)$ received platinum-containing regimens and 10 (35.7\%) etoposide-containing regimens. Seventeen of the 28 patients had a second relapse $(60.7 \%)$. Four $(23.5 \%)$ received third-line chemotherapy and all relapsed for a third time. One received fourth-line chemotherapy.

Primary sites of disease were grouped by organ system to aid analysis (table 1). The commonest primary sites were genitourinary $(\mathrm{n}=51,32.1 \%)$ and gynaecological $(\mathrm{n}=49,30.8 \%)$, followed by upper GI $(\mathrm{n}=29,18.2 \%)$, and head and neck $(\mathrm{n}=14,8.8 \%)$. Primary EPSCCs of the breast and CNS were the rarest. At diagnosis, 70 patients presented with metastatic disease and 87 with non-metastatic disease, accounting for $44.0 \%$ and $54.7 \%$ of patients, respectively. For two patients this information was unavailable $(1.3 \%)$. Only one patient was recorded as having had brain metastases at presentation $(0.6 \%)$. This was from a pancreatic primary site.

Of the 159 patients, $74(46.5 \%)$ were treated with a radical intent, $83(52.2 \%)$ palliative intent and for $2(1.3 \%)$ this information was unavailable. Fifty one $(32.1 \%)$ patients received chemotherapy only, 17 $(10.7 \%)$ radiotherapy only and $15(9.4 \%)$ had surgery only. Chemoradiotherapy was given in 30 cases $(32.1 \%)$ and surgery with preoperative or postoperative chemotherapy in $34(21.4 \%)$. Treatment details were unavailable for $12(7.5 \%)$.

Of the total 159 patients 89 relapsed (55.9\%). Twenty two patients $(13.8 \%)$ had local recurrence at first relapse, $47(29.6 \%)$ distant metastases only and $20(12.6 \%)$ had both local and distant disease. Thirty eight patients
Table 1 Primary sites of extrapulmonary small cell carcinomas identified

\begin{tabular}{ll}
\hline & Frequency (\%) \\
Site of primary cancer & [Contributing cases] \\
\hline Breast & $3(1.9)$ \\
Lower gastrointestinal & $7(4.4)$ \\
Bowel & 7 \\
Upper gastrointestinal & $29(18.2)$ \\
Liver & {$[4]$} \\
Oesophagus & {$[16]$} \\
Pancreas & {$[7]$} \\
Stomach & {$[1]$} \\
Small bowel & {$[1]$} \\
Genitourinary & $51(32.1)$ \\
Bladder & {$[30]$} \\
Prostate & {$[17]$} \\
Other & {$[4]$} \\
Gynaecological & $49(30.8)$ \\
Cervix & {$[20]$} \\
Endometrium & {$[6]$} \\
Ovary & {$[19]$} \\
Other & {$[4]$} \\
Head and neck & $14(8.8)$ \\
Pharynx & {$[1]$} \\
Parotid & {$[1]$} \\
Salivary gland & {$[3]$} \\
Other & {$[9]$} \\
Unknown primary & $6(3.8)$ \\
Lymph nodes only & {$[6]$} \\
\hline
\end{tabular}

$(23.9 \%)$ progressed on first-line treatment and died shortly after. The commonest site for metastatic disease was the liver (18 of 89 patients, $20.2 \%$ ). Only four patients had brain metastases at time of first relapse (two brain only and two extracranial and intracranial disease), representing $2.5 \%$ of all patients. There was no documentation of these patients having been symptomatic. Nineteen patients were excluded from TTDR analysis due to lack of accurate documentation of timing or sites of disease at relapse. From the remaining 140 patients assessed, median follow-up time for all patients was 5.8 months (range: 4 days-10.7 years). Sixty seven patients relapsed and 73 were censored. Median TTDR was 12.2 months (95\% CI 1.9 to 22.5 months). For the first 5 years following treatment the survival percentage was $50.7 \%, 38.3 \%, 34.6 \%$, $32.3 \%$ and $29.4 \%$, respectively. The percentage then plateaued and remained unchanged to 10 years.

PFS analysis was performed on all 159 patients. One hundred and twenty seven patients progressed or died and 32 were censored. Median PFS for all patients was 8.4 months (95\% CI 6.7 to 10.2 months) (figure 1 ). OS analysis was performed on all 159 patients. One hundred and fourteen patients died and 45 were censored. Median OS for all, non-metastatic and metastatic patients was 13.4 months (95\% CI 10.8 to 16.0$)$, 19.5 months (95\% CI 9.3 to 29.7 ) and 7.6 months (95\% CI 5.0 to 10.2), respectively (figure $2 \mathrm{~A}$, grouped by disease stage). When analysed for treatment intent, patients treated with 


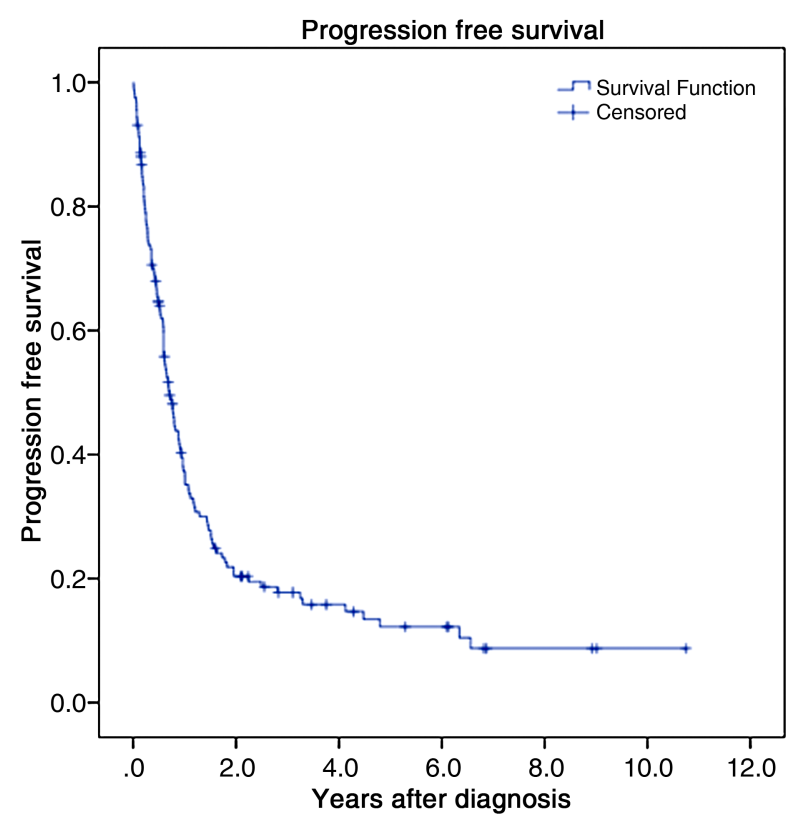

Figure 1 Kaplan-Meier plot for progression-free survival for all patients.

palliative ('non-radically treatable') versus radical intent had a poorer OS of 8.8 months (95\% CI 6.5 to 11.0) compared to 25.6 months (95\% CI 4.1 to 47.1 ), respectively (figure 2B). All variables in the univariate analysis were significantly associated with survival. OS for gynaecological, and head and neck patients, was significantly longer compared to GI patients, while for other tumour types despite a suggestion thereof, this was not significant (table 2). However, in the multivariate analysis only treatment received and metastatic stage were significant in the final model (table 3). As the core strategies for the treatment of high-grade neuroendocrine tumours have remained relatively consistent throughout the years and given the size of our data set, we did not perform differential time-to-event analysis.

\section{DISCUSSION}

We have reviewed the outcomes of one of the largest retrospective data sets of consecutive patients with EPSCC identified from two cancer centres. Our data have confirmed that EPSCC is a highly aggressive carcinoma with a poor prognosis for metastatic disease, although substantially improved for those treated radically. We confirm the common anatomical sites of primary and suggest differences in outcome from differing primary site. We confirm a high rate of relapse to distant sites, especially the liver and identify that this occurs early, suggesting a CT-based surveillance strategy might be of benefit in order to identify early asymptomatic relapsed disease. Moreover, we have documented that, unlike SCLC where up to $40.4 \%$ patients with extensive disease develop brain metastases at 1 year in the absence of PCI, ${ }^{8}$ brain metastases in EPSCC are rare
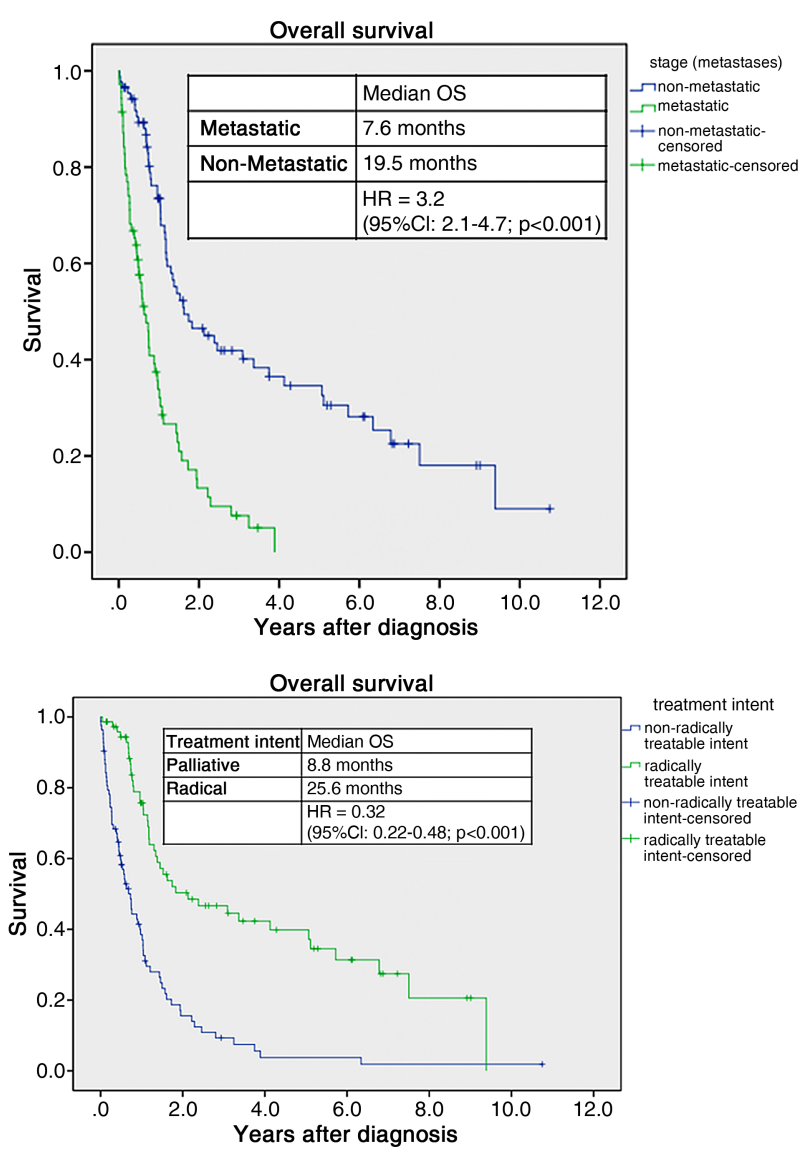

Figure 2 (A) Kaplan-Meier plot for overall survival (OS) for all patients, grouped by disease stage at diagnosis.

(B) Kaplan-Meier plot for OS for all patients, grouped by treatment intent.

(2.5\% in our study) at presentation and on follow-up. This is consistent with other retrospective data sets that have reported incidence of 4-13\%, ${ }^{13} 182024$ including a registry series $\left(6.4 \%\right.$ incidence $\left.{ }^{23}\right)$. Other differences compared to those typically observed in SCLC include a male:female ratio of 1:1.3. (compared to SCLC, $1.7: 1^{12}$ ) and a low recorded smoking history, consistent with that from other EPSCC series, with proportions of smokers ranging from $19 \%$ current and $32 \%$ ex-smokers ${ }^{13}$ to $30 \%$ current smokers. ${ }^{23}$

Data on outcomes and natural history of EPSCC have been limited given its rarity, and generally based on smaller retrospective case series to date (table 4), barring two registry series, one from South East England $^{12}$ and one from Ireland. ${ }^{23}$ Our data set is one of the largest consecutive patient series reported, and presents outcomes consistent with other data sets.

Specifically, the commonest primary sites of disease in our study were genitourinary and gynaecological, followed by upper GI, and head and neck. Other studies have shown very similar findings, ${ }^{12-15} 182325$ with the exception of Wong et al, ${ }^{12}$ where breast was the primary site in $10 \%$ of cases. In agreement with previous studies primary site of disease is associated with OS. From previous data sets, patients with GI primaries have the worst 
Table 2 Univariate Cox regression analysis for overall survival

\begin{tabular}{|c|c|c|c|}
\hline Variables & HR & $95 \% \mathrm{Cl}$ & p Value \\
\hline \multicolumn{4}{|l|}{ Stage } \\
\hline Non-metastatic & 1 & & \\
\hline Metastatic & 3.2 & 2.1 to 4.7 & $<0.001$ \\
\hline \multicolumn{4}{|l|}{ Treatment intent } \\
\hline Non-radically treatable intent & 1 & & \\
\hline Radically treatable intent & 0.3 & 0.2 to 0.5 & $<0.001$ \\
\hline \multicolumn{4}{|l|}{ Site of primary } \\
\hline Lower/upper gastrointestinal & 1 & & \\
\hline Genitourinary & 0.7 & 0.4 to 1.2 & 0.177 \\
\hline Gynaecological & 0.6 & 0.3 to 0.9 & 0.027 \\
\hline Head and neck & 0.3 & 0.2 to 0.8 & 0.008 \\
\hline Breast & 0.3 & 0.04 to 2.1 & 0.218 \\
\hline Variable overall & & & 0.030 \\
\hline \multicolumn{4}{|c|}{ Treatment received (prior to progression) } \\
\hline No treatment received (reference) & 1 & & \\
\hline Chemotherapy & 0.05 & 0.02 to 0.12 & $<0.001$ \\
\hline Radiotherapy & 0.05 & 0.02 to 0.14 & $<0.001$ \\
\hline Surgery & 0.02 & 0.01 to 0.05 & $<0.001$ \\
\hline Combination chemoradiotherapy & 0.03 & 0.01 to 0.07 & $<0.001$ \\
\hline Combination chemosurgery & 0.02 & 0.01 to 0.04 & $<0.001$ \\
\hline Variable overall & & & $<0.001$ \\
\hline
\end{tabular}

prognosis, $^{11} 1217$ with breast and genitourinary sites reporting improved survival. ${ }^{11} 12142225$ These findings can be in part explained by the disease stage at diagnosis. ${ }^{13} 141718$ In our study, although site was a significant covariate of survival, likely due to limited numbers of patients, genitourinary, and head and neck patients had significantly better survival compared to GI patients. These differences in outcomes change by site in our report and are influenced by numbers of cases of each anatomical location identified, which in turn likely reflects local referral patterns.

Median OS in this study was 13.4, 7.6 and 19.5 months for all, metastatic and non-metastatic patients, respectively, again relatively consistent with previous studies identifying an OS of 9.8-14 months, 2-9.2 months and 16.8-34 months for all, extensive-stage and limited-stage patients, respectively (table 4). ${ }^{11-14} 2021$ Wong et al reported an overall 3-year survival of $30 \%$ for patients presenting with limited disease and $10 \%$ for those with extensive disease, comparable to the $34.6 \%$ survival for all patients at 3 years in this study.

In the multivariate analysis only treatment intent and stage were significant covariates, again consistent with existing data sets. ${ }^{1920}$ Other studies have found a higher white cell count at diagnosis, ${ }^{14}$ poor performance status, weight loss prior to diagnosis, omission of radical radiotherapy $^{11} 13^{14}{ }^{24}$ and male gender ${ }^{25}$ to be significantly associated with a poor OS. A better outcome for female patients could in part be attributed to the early stage at diagnosis of gynaecological EPSCC. ${ }^{14} 25$ The same factors are important in regard to PFS, which has been reported as 13.5-20 months in limited and 3-12 months in extensive disease in other studies. ${ }^{14} 20$

The studies included in this table are not the result of a systematic review. These are the largest studies on EPSCC with OS data on patients with LS and ES.

Table 3 Multivariate Cox regression model for overall survival

\begin{tabular}{|c|c|c|c|}
\hline Variables & HR & $95 \% \mathrm{Cl}$ & p Value \\
\hline \multicolumn{4}{|l|}{ Stage } \\
\hline Non-metastatic & 1 & & \\
\hline Metastatic & 2.4 & 1.5 to 3.8 & $<0.001$ \\
\hline \multicolumn{4}{|l|}{ Treatment received (prior to progression) } \\
\hline No treatment received (reference) & 1 & & \\
\hline Chemotherapy & 0.06 & 0.02 to 0.13 & $<0.001$ \\
\hline Radiotherapy & 0.09 & 0.03 to 0.23 & $<0.001$ \\
\hline Surgery & 0.03 & 0.01 to 0.08 & $<0.001$ \\
\hline Combination chemoradiotherapy & 0.04 & 0.02 to 0.10 & $<0.001$ \\
\hline Combination chemosurgery & 0.02 & 0.01 to 0.06 & $<0.001$ \\
\hline Variable overall & & & $<0.001$ \\
\hline
\end{tabular}


Table 4 Summary of overall survival for patients with extrapulmonary neuroendocrine carcinoma (EPSCC; in months) in major studies reported

\begin{tabular}{|c|c|c|c|c|}
\hline \multirow[b]{2}{*}{ Study } & \multirow[b]{2}{*}{ Number of patients (LS and ES) } & \multicolumn{3}{|c|}{ Median overall survival (months) } \\
\hline & & All patients & Limited stage & Extensive stage \\
\hline Brennan et al ${ }^{13}$ & $120(84$ and 36$)$ & - & 16.8 & 8.4 \\
\hline Cicin et $a P^{0}$ & 11 (3 and 8) & 10 & 17 & 5 \\
\hline Current study & 159 (87 and 70$)$ & 13.4 & 19.5 & 7.6 \\
\hline Dakhil et $a{ }^{26}$ & 35 (20 and 15) & - & 36 & 5 \\
\hline Haider et al ${ }^{14}$ & 101 (51 and 50) & 9.38 & 34 & 2 \\
\hline Kim et $a l^{18}$ & $34(23$ and 11$)$ & 14 & 19.8 & 7 \\
\hline Naidoo et $a P^{3}$ & $288(65 \text { and } 186)^{*}$ & - & 15.2 & 2.3 \\
\hline Terashima et $a{ }^{21}$ & $41(0$ and 41$) \dagger$ & 9.2 & 0 & 9.2 \\
\hline Wong et $a 1^{12}$ & 1618 (532 and 682)‡ & - & 12 & 3.4 \\
\hline
\end{tabular}

While a number of biases may have influenced our results given the retrospective nature of this study and lack of central pathology verification, our data support those identified from other, smaller data sets. We have shown that while similar to SCLC in terms of high response rates to platinum-etoposide-based chemotherapy, and high rates of distant metastases (especially to the liver), there are notable differences to ESPCC. Here, incidence in smokers is lower than SCLC and may potentially reflect differing pathobiology. Moreover, the brain remains an uncommon site of metastases and we therefore do not recommend PCL. Finally, the site of primary may influence prognosis, and survival is optimal with a radical strategy. ESPCC remains a rare diagnosis, and concerted efforts into better understanding the biological mechanisms that underpin its pathogenesis and relationship to SCLC pathobiology is urgently warranted in order to improve clinical outcomes.

Contributors SP designed the study. RG participated in the design of the study and performed the statistical analysis. SG, JN, SS, RC, TB, AW and SP were involved in patient identification, and data collection and analysis. SG and SP participated in writing the manuscript. All authors read and approved the final manuscript.

Funding SG, JN, SS, RG, AW and SP acknowledge NHS funding to the NIHR Biomedical Research Centre.

Competing interests None.

Provenance and peer review Not commissioned; externally peer reviewed.

Data sharing statement Extra data can be accessed via the Dryad data repository at http://datadryad.org/ with the doi:10.5061/dryad.t2q91.

Open Access This is an Open Access article distributed in accordance with the Creative Commons Attribution Non Commercial (CC BY-NC 4.0) license, which permits others to distribute, remix, adapt, build upon this work noncommercially, and license their derivative works on different terms, provided the original work is properly cited and the use is non-commercial. See: http:// creativecommons.org/licenses/by-nc/4.0/

\section{REFERENCES}

1. Klimstra DS, Modlin IR, Coppola D, et al. The pathologic classification of neuroendocrine tumors: a review of nomenclature, grading, and staging systems. Pancreas 2010;39:707-12.
2. Hirabayashi K, Zamboni G, Nishi T, et al. Histopathology of gastrointestinal neuroendocrine neoplasms. Front Oncol 2013;3:2.

3. van Meerbeeck JP, Fennell DA, De Ruysscher D. Small-cell lung cancer. Lancet 2011;378:1741-55

4. Govindan R, Page N, Morgensztern D, et al. Changing epidemiology of small-cell lung cancer in the United States over the last 30 years: analysis of the surveillance, epidemiologic, and end results database. J Clin Oncol 2006;24:4539-44.

5. Varghese AM, Zakowski MF, Yu HA, et al. Small-cell lung cancers in patients who never smoked cigarettes. J Thorac Oncol 2014:9:892-6.

6. Wolfson AH, Bae K, Komaki R, et al. Primary analysis of a phase II randomized trial Radiation Therapy Oncology Group (RTOG) 0212 impact of different total doses and schedules of prophylactic cranial irradiation on chronic neurotoxicity and quality of life for patients with limited-disease small-cell lung cancer. Int J Radiat Oncol Biol Phys 2011;81:77-84.

7. Foster NR, Qi $\mathrm{Y}$, Shi $\mathrm{Q}$, et al. Tumor response and progression-free survival as potential surrogate endpoints for overall survival in extensive stage small-cell lung cancer: findings on the basis of North Central Cancer Treatment Group trials. Cancer 2011;117:1262-71.

8. Slotman B, Faivre-Finn C, Kramer G, et al. Prophylactic cranial irradiation in extensive small-cell lung cancer. $N$ Engl J Med 2007;357:664-72.

9. Prophylactic Cranial Irradiation Overview Collaborative Group. Cranial irradiation for preventing brain metastases of small cell lung cancer in patients in complete remission. Cochrane Database Syst Rev 2000;(4):CD002805.

10. Duguid JB, Kennedy AM. Oat-cell tumours of mediastinal glands $J$ Pathol Bacteriol 1930:33:93-9.

11. Kim KO, Lee HY, Chun SH, et al. Clinical overview of extrapulmonary small cell carcinoma. J Korean $\mathrm{Med} \mathrm{Sci}$ 2006;21:833-7.

12. Wong YNS, Jack RH, Mak $\mathrm{V}$, et al. The epidemiology and survival of extrapulmonary small cell carcinoma in South East England, 19702004. BMC Cancer 2009;9:209.

13. Brennan SM, Gregory DL, Stillie A, et al. Should extrapulmonary small cell cancer be managed like small cell lung cancer? Cancer 2010;116:888-95

14. Haider K, Shahid RK, Finch D, et al. Extrapulmonary small cell cancer: a Canadian province's experience. Cancer 2006;107:2262-9.

15. Huang TL, Huang $\mathrm{CH}$, Tang $\mathrm{Y}$, et al. Extrapulmonary small cell carcinoma-a medical center's experience. Chang Gung Med $J$ 2006;29:590-5.

16. Frazier SR, Kaplan PA, Loy TS. The pathology of extrapulmonary small cell carcinoma. Semin Oncol 2007;34:30-8.

17. Brenner B, Tang LH, Klimstra DS, et al. Small-cell carcinomas of the gastrointestinal tract: a review. J Clin Oncol 2004;22:2730-9.

18. $\mathrm{Kim} \mathrm{JH}$, Lee SH, Park J, et al. Extrapulmonary small-cell carcinoma: a single-institution experience. Jpn J Clin Oncol 2004;34:250-4.

19. Ochsenreither S, Marnitz-Schultze S, Schneider A, et al. Extrapulmonary small cell carcinoma (EPSCC): 10 years' multi-disciplinary experience at Charité. Anticancer Res 2009;29:3411-15. 
20. Cicin I, Karagol H, Uzunoglu S, et al. Extrapulmonary small-cell carcinoma compared with small-cell lung carcinoma: a retrospective single-center study. Cancer 2007;110:1068-76.

21. Terashima T, Morizane C, Hiraoka N, et al. Comparison of chemotherapeutic treatment outcomes of advanced extrapulmonary neuroendocrine carcinomas and advanced small-cell lung carcinoma. Neuroendocrinology 2012;96:324-32.

22. Hoskins PJ, Swenerton KD, Pike JA, et al. Small-cell carcinoma of the cervix: fourteen years of experience at a single institution using a combined-modality regimen of involved-field irradiation and platinum-based combination chemotherapy. J Clin Oncol 2003;21:3495-501.
23. Naidoo J, Teo MY, Deady S, et al. Should patients with extrapulmonary small-cell carcinoma receive prophylactic cranial irradiation? J Thorac Oncol 2013;8:1215-21.

24. Früh M, Kacsir B, Ess S, et al. Extrapulmonary small cell carcinoma: an indication for prophylactic cranial irradiation? A single center experience. Strahlenther Onkol 2011;187:561-7.

25. Lin YL, Chung CY, Chang CS, et al. Prognostic factors in extrapulmonary small cell carcinomas. A large retrospective study. Oncology 2007;72:181-7.

26. Dakhil CSR, Wick JA, Kumar AKL, et al. Extrapulmonary small cell carcinoma: the University of Kansas experience and review of literature. Med Oncol 2014;31:187. 